\title{
Legitimation des Gewaltmonopols
}

\section{Klar Unklares}

Das Gewaltmonopol des Staates wird meist vorausgesetzt und apologetisch verwendet als Ausdruck eines zivilisatorischen Fortschritts. In der juristischen Diskussion wird es eher ,irgendwie“ als spezifisch in Verbindung gebracht mit dem Rechtsstaat, manchmal mit der Souveränitätslehre oder mit dem Auftrag des Staates, einen Friedenszustand herzustellen. In einem eigentlich ziemlich guten Kommentar zum Grundgesetz heißt es etwa zu Art. 20: „Andererseits folgt aus dem Rechtsstaatsprinzip in Korrespondenz zum Gewaltmonopol die Pflicht des Staates, eine funktionsfähige Strafjustiz zu gewährleisten...." Ähnlich nebulös klingt das folgende Zitat aus demselben Kommentar: „Bezugspunkt der Gewaltenteilung sind die Freiheitsrechte der Individuen, vor denen sich, als Korrelat zum Gewaltmonopol des Staates, die Ausübung politischer Herrschaft rechtfertigen muss...."2 Das verstehe wer will, ich kann daraus allenfalls die Aufforderung zu freier Assoziation in einer tiefenpsychologischen Sitzung entnehmen oder das Bemühen, das Gewaltmonopol doch auch mal zu erwähnen. Interessant ist, dass der Autor das Gewaltmonopol und die Gewaltenteilung in einem Satz erwähnt, ohne zu bemerken, dass hier zumindest begrifflich ein Spannungsverhältnis besteht, das der Aufklärung bedarf, wenn man bei den Lesern nicht voraussetzt, dass sie es eh schon wissen - dann aber kann man sich solche Bücher auch sparen. Diese oberflächliche Diskussion des Gewaltmonopols ist durchaus symptomatisch. Klaus Stern bringt es fertig, auf 6000 Seiten zum „Staatsrecht der Bundesrepublik Deutschland“ das Gewaltmonopol ähnlich en passant zu behandeln wie der Autor der oben zitierten Kommentierung.

Wenn Gewaltmonopol und Gewaltenteilung sich nicht widersprechen sollen, müssen offensichtlich zwei unterschiedliche Begriffe von „Gewalt“ verwendet werden. Auch das ist nicht ungewöhnlich. Das Strafrecht schafft es - trotz des Gebots der systematischen Auslegung, das verlangt, einem Begriff zumindest innerhalb desselben Gesetzes eine identische Bedeutung zu geben - wohl insgesamt vier Gewaltbegriffe ins Strafgesetzbuch hineinzulesen. Die Gewaltenteilung bezieht sich auf die Verteilung von Machtressourcen auf unterschiedliche Staatsorgane, nicht dagegen auf die Anwendung von körperlichem Zwang.

\section{Definitiönchen}

Damit ist eine Unterscheidung eingeführt, die Max Weber in seiner berühmten Definition des Staates verwendet: "Staat soll ein politischer Anstaltsbetrieb heißen, wenn und insoweit sein Verwaltungsstab erfolgreich das Monopol legitimen physischen Zwangs

1 Schulze-Fielitz, H.: in Dreier, H.: GG-Kommentar Bd.II, Art. 20, Rnr. 214.

2 Schulze-Fielitz, H.: in Dreier, H.: GG-Kommentar Bd.II, Art. 20, Rnr. 8. 
für die Durchführung der Ordnung in Anspruch nimmt."3 Das Gewaltmonopol meint das Monopol auf die Anwendung physischen Zwangs. Lassen wir die anderen Elemente der Definition noch für einen Augenblick beiseite. Das Monopol des Staates ist in dieser Lesart offenbar mit der Verteilung auf unterschiedliche Machtzentren im Staat vereinbar - das Monopol besitzt der Staat, innerhalb des Staates aber - wenn man Gewaltenteilung zumindest rudimentär voraussetzt - nicht ein Zentrum, eine monokratische Institution. Hier wird schon begrifflich sichtbar, dass die Bezeichnung Monopol ein Problem darstellt. Das Monopol lässt sich ableiten vom griechischen Wort „monos“, das mit einzig, allein und einsam übersetzt wird - das jeder im Unterschied zu stereo kennt -, aber im Wort Monokratie ebenfalls untergebracht ist. Dieses begriffliche Problem ist kein Zufall, es war so gemeint wie eine ideengeschichtliche Betrachtung zeigen wird: die Gewalt sollte monopolisiert werden, nämlich beim Monarchen - worin monos ebenfalls steckt.

Vor dieser Betrachtung will ich aber noch einen Blick auf die implizit getroffene Unterscheidung zwischen Gewalt und Macht wagen. Macht kommt im juristischen Sprachgebrauch merkwürdigerweise so gut wie nicht vor. Eine Ausnahme bildet $\S 93$ StGB, wonach Staatsgeheimnisse nicht an eine „fremde Macht“ verraten werden dürfen. Ansonsten gibt es Kombinationen mit Vollmacht, aber - soweit ich sehe - die Macht nicht allein. Sie scheint außerjuristisch zu sein oder allenfalls zwischenstaatlich eine Rolle zu spielen. Für Macht ist ebenfalls Webers Definition berühmt geworden: „Macht bedeutet jede Chance, innerhalb einer sozialen Beziehung den eigenen Willen auch gegen Widerstreben durchzusetzen, gleichviel worauf diese Chance beruht. Herrschaft soll heißen die Chance, für einen Befehl bestimmten Inhalts bei angebbaren Personen Gehorsam zu finden."4 Weber grenzt Macht von Herrschaft ab, aber - soweit ich sehe - nicht von Gewalt. Juristen beschäftigen sich mit Herrschaft, nicht mit Macht, folgen also gleichsam Webers Definition.

Die Unterscheidung von Macht und Gewalt findet sich in einer überzeugenden $\mathrm{Ab}$ grenzung bei Kant: „Ein Gegenstand meiner Willkür aber ist das, wovon beliebigen Gebrauch zu machen ich das physische Vermögen habe, dessen Gebrauch in meiner Macht (potentia) steht: wovon noch unterschieden werden muss, denselben Gegenstand in meiner Gewalt (in potestatem meam redactum) zu haben, welches nicht bloß ein Vermögen, sondern auch einen Akt der Willkür voraus setzt."5 Gewalt ist also eine Handlungsform der Macht, sie umfasst die Macht in ihrer Potenz und den Willen, diese in einem Handlungsakt einzusetzen. Gewalt meint das Vermögen zur Durchführung einer Handlung, durch die auf Menschen bzw. Gegenstände beeinflussend, verändernd und/oder schädigend eingewirkt wird. Nehmen wir Webers Staatsdefinition noch hinzu, dann handelt es sich bei Gewalt um einen Akt der physischen Einwirkung.

Die Macht ist offenkundig umfassender als Gewalt. Sie wird etwa von Arendt, Foucault oder Habermas in ganz unterschiedlicher Weise vor allem in der menschlichen Interaktion oder in Diskursen verortet. Macht ist in der Gesellschaft unterschiedlich verteilt. Macht findet man auch in der Direktionsbefugnis des Unternehmers gegenüber seinen Arbeitern. Es wird also akzeptiert, dass Macht z.B. dem Eigentum entspringt nicht aber Gewalt im Sinne von physischem Zwang. Weiter formuliert das Postulat der

3 Weber, M.: Wirtschaft und Gesellschaft, S. 29.

4 Weber, M.: Wirtschaft und Gesellschaft, Kap. I, § 16.

5 Kant, I.: Die Metaphysik der Sitten, Kant-Werke Bd. 8, S. 354. 
Gewaltenteilung als Norm, dass Macht innerhalb des Staates auf unterschiedliche Institutionen verteilt ist. Das Gewaltmonopol als normatives Postulat - und darüber müssen wir ja reden, wenn wir von der Legitimation und nicht wie Weber in der Staatsdefinition nur von der Empirie reden - fordert dagegen, dass Gewalt nur vom Staat eingesetzt werden darf, nicht in unterschiedlicher Weise in der Gesellschaft, bei Personen oder Institutionen außerhalb des Staates verteilt sein soll.

\section{Gewalt-Monopol und Staat}

Daraus ergeben sich wiederum drei Probleme. Erstens werden regelmäßig die Ausnahmen genannt wie die Notwehr- oder Selbsthilferechte. ${ }^{6}$ Zweitens darf ja keineswegs „der Staat“ insgesamt, also alle Institutionen des Staates Gewalt anwenden, sondern nur spezifische Organe, die dazu ermächtigt sind. Gewalt darf die Polizei einsetzen, nicht aber der Hochschullehrer. Drittens stellt sich die Frage: Was ist denn nun wieder der Staat in Abgrenzung von der Gesellschaft oder nur vom Nicht-Staat. Fangen wir mit letzterem an: Webers Definition hilft hier nicht weiter, weil er den Staat ja gerade durch das Gewaltmonopol definiert - wir wollen aber gerade normativ wissen, wer dieses Monopol in Anspruch nehmen darf. Weiter hilft auch in diesem Fall nicht Jellineks DreiElementen-Lehre: sie ist nicht nur unterkomplex, sondern definiert den Staat ebenfalls durch die Staatsgewalt.

Kants berühmte Definition lautet: „Ein Staat (civitas) ist die Vereinigung einer Menge von Menschen unter Rechtsgesetzen. " Und Hans Kelsen formuliert: "Die Erkenntnis des Staates kann sich seines Wesens nicht anders bemächtigen, als indem sie dieses soziale Gebilde als eine Ordnung menschlichen Verhaltens begreift. Nähere Untersuchung zeigt, dass es eine soziale Zwangsordnung ist und dass diese Zwangsordnung mit der Rechtsordnung identisch sein muss, da es dieselben Zwangsakte sind, die beide charakterisieren... Der Staat ist eine Rechtsordnung." 8 Diese Rechtsordnung müsse "gewisse arbeitsteilig funktionierende Organe" ausgebildet und einen "gewissen Grad von Zentralisation" erreicht haben. ${ }^{9}$ Nicos Poulantzas definiert den Staat zusammenfassend folgendermaßen: "Es handelt sich um einen zentralisierten, spezialisierten Apparat von spezifisch politischer Natur; er besteht aus einer Zusammenfügung von anonymen, unpersönlichen und formell von der ökonomischen Macht unterschiedenen Funktionen, deren Verknüpfung auf einer Axiomatisierung von Gesetzen und Regeln, durch die Tätigkeitsbereiche verteilt werden, und von Kompetenzen sowie auf einer auf das VolkNation gegründeten Legitimität basiert."10

6 Gusy, Ch.: Rechtsgüterschutz als Staatsaufgabe, in: DöV 1996, S. 573/576; Hammer, F.: Private Sicherheitsdienste, staatliches Gewaltmonopol, Rechtsstaatsprinzip und „schlanker Staat", in: DöV 2000, S. 613/615.

7 Kant, Metaphysik der Sitten, § 45, S. 431.

8 Kelsen, H.: Reine Rechtslehre, S. 117; dazu: Reisinger, Der Staatsbegriff Kelsens, in: Krawietz u.a., Ideologiekritik, S. $483 \mathrm{ff}$.

9 Kelsen, H.: Reine Rechtslehre, S. 117 f.

10 Poulantzas, N.: Staatstheorie, S. 81. 
Diese Staatsbegriffe lassen sich selbstverständlich kritisch hinterfragen, ${ }^{11}$ was hier nicht unsere Aufgabe ist. Sie haben bei allen Unterschieden eine Gemeinsamkeit: Der Staat ist untrennbar verknüpft mit seinen Gesetzen. Dann folgt: Die Gesetze bilden die Grundlage für die Gewaltanwendung, sie legitimieren das Gewaltmonopol. Akzeptiert man das zunächst, gibt es auch kein Problem mehr mit den Notwehr- und Selbsthilferechten und mit der Zuordnung des Gewaltmonopols zu bestimmten Staatsorganen. Die Gesetze legitimieren sowohl die Gewaltanwendung durch spezifische, keineswegs alle staatliche Organe wie in bestimmten Fällen auch durch Private. Das heißt weiter für die folgenden Überlegungen, dass nach der Legitimation für die Rechtsordnung insgesamt und nicht nur für das Gewaltmonopol gesucht werden muss.

\section{Gewalt-Monopol und Recht}

Dieses Zwischenergebnis ist möglicherweise kontraintuitiv. Gibt es nicht Staaten, Diktaturen oder autoritäre Regime, die sich eben nicht auf das Recht stützen, sondern auf die Bajonette? Sind das nicht trotzdem Staaten und nehmen diese Staaten nicht möglicherweise das Gewaltmonopol besonders effektiv in Anspruch? Der Einwand greift nur prima facie, denn: erstens unterscheiden sich Staaten, die sich nur auf ihre Bajonette stützen, nicht von besonders effektiven Räuberbanden im Großen. Franz Neumann hat den NS-Staat als Unstaat oder Nichtstaat, als Behemoth charakterisiert, weil es kein berechenbares Recht gegeben habe. Es habe eine Menge technischer Regeln gegeben, aber kein Rechtssystem, das diesen Namen verdient habe. Die NS-Gesetze könnten nur als Recht bezeichnet werden, wenn „Gesetz nichts weiter ist als der Wille des Souveräns“, aber diese Bezeichnung hätten sie nicht verdient, wenn „Gesetz im Gegensatz zum Befehl des Souveräns entweder der Form oder dem Inhalt nach rational sein muss. Das nationalsozialistische Rechtssystem ist nichts anderes als eine Technik der Manipulation der Massen durch Terror." Das System sei deshalb als Unstaat zu bezeichnen, weil es einen ,Zustand vollkommener Gesetzlosigkeit“ darstelle. ${ }^{12}$

Das klingt ziemlich rechtsgläubig, gleichsam deutsch-rechtstreu, stimmt aber im Ergebnis mit Webers Definition überein. Den Staat charakterisiert er nicht durch das Gewaltmonopol, sondern durch „das Monopol legitimen physischen Zwangs“ - die Legitimität ist in das Gewaltmonopol eingelassen, denn selbstverständlich wird auch von der Mafia physischer Zwang vergleichsweise erfolgreich ausgeübt, aber eben nicht legitim. Die Legitimität, nach der wir ja suchen, gründet in der Gesetzmäßigkeit der Gewaltausübung, die Legitimität letzterer ist dann natürlich weiter zu prüfen. Aber ohne Recht, so lässt sich folgern, keine legitime Gewaltausübung. Das heißt nun nicht, dass es nicht Diktaturen oder Staaten mit ungerechtem Recht gibt, die sich erfolgreich auf das Gewaltmonopol stützen. Deren Legitimität ist zu prüfen. Es heißt zunächst, dass das Gewaltmonopol an das Recht geknüpft ist.

Diese These lässt sich zweitens auch begründen, wenn man Kants Begriff des Rechts folgt. Das Recht definiert er als den ,Inbegriff derjenigen Bedingungen, unter denen die Willkür des einen mit der Willkür des andern nach einem allgemeinen Gesetze der

11 Dazu ausführlich: Fisahn, A.: Herrschaft im Wandel (Köln 2008), passim.

12 Neumann, F.: Behemoth (Frankfurt 1977), S. 530 ff. 
Freiheit zusammen vereinigt werden kann.“ Daraus folgt dann ziemlich logisch, dass ,alles, was Unrecht ist, ein Hindernis der Freiheit nach allgemeinen Gesetzen (ist); der Zwang aber ist ein Hindernis oder Widerstand, der der Freiheit geschieht. Folglich: wenn ein gewisser Gebrauch der Freiheit selbst ein Hindernis der Freiheit nach allgemeinen Gesetzen (d.i. unrecht) ist, so ist der Zwang, der diesem entgegengesetzt wird, als Verhinderung eines Hindernisses der Freiheit mit der Freiheit nach allgemeinen Gesetzen zusammen stimmend, d.i. recht: mithin ist mit dem Rechte zugleich eine Befugnis, den, der ihm Abbruch tut, zu zwingen, nach dem Satze des Widerspruchs verknüpft." Der Zwang, d.h. die Ausübung des Gewaltmonopols gegenüber dem Rechtsbrecher, schränkt zwar die individuelle Freiheit des Rechtsbrechers ein, stellt aber mit dem Recht die allgemeine Freiheit wieder her. Deshalb folgert er weiter: „,Das Recht darf nicht als aus zwei Stücken, nämlich der Verbindlichkeit nach einem Gesetze und der Befugnis dessen, der durch seine Willkür den andern verbindet, diesen dazu zu zwingen, zusammengesetzt gedacht werden, sondern man kann den Begriff des Rechts in der Möglichkeit der Verknüpfung des allgemeinen wechselseitigen Zwanges mit jedermanns Freiheit unmittelbar setzen.“ Oder kurz: „Recht und Befugnis zu zwingen bedeuten also einerlei."13 Bei einer Gleichung kann man logischerweise auch den umgekehrten Schluss ziehen. So erklärt sich möglicherweise die „Korrespondenz“ zwischen Rechtsstaat und Gewaltmonopol, die oben aus dem GG-Kommentar zitiert wurde.

Recht impliziert Gewalt - diesen Satz kann man wie etwa Walter Benjamin auch kritisch lesen oder wie Jaque Derrida durch kritische Dekonstruktion als Resultat gewinnen. Was folgt? Möglicherweise ein radikaler Rechts- und Gewaltskeptizismus, letztlich die Negation der Möglichkeit, das Gewaltmonopol und mit diesem auch den Staat zu rechtfertigen. Wir behalten das an dieser Stelle als Merkposten, es wird wieder auftauchen.

\section{Hauptsache Ordnung}

Nun gibt es aber einen breiten Meinungsstrom, der die Legitimität des Gewaltmonopols der Legitimität des Rechts voranstellt, i.d.S. dass eine vorgängige Basislegitimation des Staates und des Gewaltmonopols aus der Friedenssicherung konstruiert wird. Die Argumentationsfolie dafür lieferte Thomas Hobbes, den ich hier nicht ausführlich referieren muss. Der Krieg aller gegen alle im Naturzustand führt schließlich zu einem Vertragsabschluss, der als Unterwerfungsvertrag und gleichzeitig als Austauschvertrag konzipiert ist. Ausgetauscht wird Frieden oder Sicherheit gegen die Unterwerfung unter den Willen des Monarchen. Der Vertrag ist zu denken als Akt, in dem ein ,jeder zu einem jeden sagte: ,Ich übergebe mein Recht, mich selbst zu beherrschen, diesem Menschen oder dieser Gesellschaft unter der Bedingung, dass du ebenfalls dein Recht über dich ihm oder ihr abtrittst.' Auf diese Weise werden alle einzelnen eine Person und heißen Staat oder Gemeinwesen. So entsteht der große Leviathan. "14 Hobbes rechtfertigt die absolute Monarchie und das Gewaltmonopol trotz des Selbstbestimmungsrecht des

13 Kant, I: Die Metaphysik der Sitten, Kant-Werke Bd. 8, S. 337 ff.

14 Hobbes, Th.: Leviathan, S. 155. 
Menschen nur mit ihrer Frieden sichernden Funktion. ${ }^{15}$ Er rechtfertigt nicht abstrakte Staatlichkeit, sondern die bedingungslose Unterwerfung unter den Monarchen. Das Gewaltmonopol, das mit dem Unterwerfungsvertrag verbunden ist, rechtfertigt er als zivilisatorischen Fortschritt gegenüber der unkontrollierten Gewalt, der Fehde und dem Recht des Stärkeren.

Aber die Rechtfertigung bleibt erstens offenkundig auf halbem Wege stecken. Wenn Hobbes grundsätzlich die Zustimmung der Herrschaftsunterworfenen zum Gesellschaftsvertrag voraussetzt, führt dies denknotwenig zu einer Ausweitung der Zustimmungserfordernisse über die Konstituierung der öffentlichen Gewalt auf die Akte der öffentlichen Gewalt. Zweitens ist die Ambivalenz der Negation des Rechts des Stärkeren zu betonen: Mit der Unterwerfung wird der Stärkere ein für alle mal festgeschrieben und dieser Stärkere nimmt für sich auch noch das Recht in Anspruch. Das kann für die Herrschaftsunterworfenen unangenehme Folgen haben - der starke Staat ist auch für die Schwachen oder vielleicht gerade für die Schwachen, die auf Möglichkeiten der solidarischen Vereinigung angewiesen sind, keineswegs ein Segen, selbst dann nicht, wenn er an Recht gebunden ist. Die Rechtsform, das allgemeine Gesetz, gewährleistet nur ein Minimum an Freiheit über die Berechenbarkeit staatlicher Unterdrückung, schließt diese aber keineswegs aus. Anders gesagt: Der nur formal definierte Rechtsstaat allein kann das Gewaltmonopol nicht rechtfertigen. Die Negation der unkontrollierten Gewalt kann eben eine kontrollierte Gewaltordnung hervorbringen, die für die ihr Ausgelieferten mindestens ebenso unangenehm ist wie die Anarchie der Gewaltpotenziale.

Zwischenbemerkung: Das scheint dem Ausschluss des Un-Staates, der durch die Nichtexistenz von Recht gekennzeichnet ist, aus dem Kreis derjenigen, die das Gewaltmonopol in Anspruch nehmen können, zu widersprechen. Es scheint aber nur so, denn die Selbstbindung der staatlichen Gewalt durch das Recht erzeugt zwar minimale Freiräume für die Rechtsunterworfenen, aber eben nur minimale. Auch wenn man über die Rechtsform bestimmte Unterdrückungsmaschinen ganz aus dem Staatsbegriff herausnimmt, bleibt auch für die Staaten als Rechtsordnung genügend Unterdrückungspotenzial - gerade über die Monopolisierung der Gewalt. Es bleibt ein Macht- und Gewaltpotenzial, das sich nicht allein über die Monopolisierung rechtfertigen lässt.

Dennoch versuchen auch zeitgenössische Theorien, Hobbes Rechtfertigungsmodus für das Gewaltmonopol mit dem Demokratiegebot des GG, der demokratischen Legitimation, in welcher Weise auch immer zu verbinden. Die Legitimation kann dann nur zweistufig erfolgen. Auf der ersten Stufe wird abstrakte Staatlichkeit allerdings inklusive Gewaltmonopol durch die Friedens- und Ordnungssicherung gerechtfertigt. Dieser Basislegitimation wird dann die Legitimation des konkreten Staates und seines Rechts gleichsam oben aufgepfropft. Forsthoff konstruiert ein vorgelagertes Wesen des Staates gleichsam als Substanz, als dem verfassten Staat vorausgehende Entität, als geschichtliches Kontinuum, oder als gegebene Wesenheit, deren Ausgestaltung geschichtlichen Prozessen, unterschiedlichen Einflüssen und Kräften nicht unterliegt. Charakteristisch

15 Hobbes definiert dann den Staat folgerichtig über die Friedensstiftung: „Staat ist eine Person, deren Handlungen eine große Menge Menschenkraft der gegenseitigen Verträge eines jeden mit einem jeden als ihre eigenen ansehen, auf dass diese nach ihrem Gutdünken die Macht aller zum Frieden und zur gemeinschaftlichen Verteidigung anwende.“ (Hobbes, Leviathan, S. 155 f.). 
für dieses Grundverständnis ist ein Satz wie: "Die Staatsgewalt als solche ist in allen Verfassungen gleich."16

Böckenförde greift in dieser Tradition für seine Bestimmung des Staatszwecks die Definition des Politischen über die Freund-Feind-Unterscheidung von Carl Schmitt ${ }^{17}$ auf, zivilisiert sie jedoch, indem sie der Friedensfunktion des Staates subsumiert wird. Innerhalb des Staates werde die Existenz von Freund-Feind-Gruppierungen ausgeschlossen, weil staatlicherseits die friedliche, nicht-gewaltsame Lösung von Konflikten garantiert werde - nämlich durch das Gewaltmonopol. ${ }^{18}$ Der Staat wird so der Demokratie bei Böckenförde gleichsam als "Rohling" der konkreten Staatsform vorgelagert. Die konkrete, geschichtliche Erscheinung des Staates wird zur abstrakten Staatlichkeit, die unabhängig von der Form oder dem Gebrauch oder Missbrauch der staatlichen Macht affirmativ besetzt wird. Böckenförde wörtlich: "Staatlichkeit im modernen Sinn bedeutet zunächst die Organisation einer Gesellschaft als Friedenseinheit, als zu eigenen Entscheidungen befähigte Handlungseinheit und als Machteinheit."19 Diese abstrakte Staatlichkeit kann nur gedacht werden als Einheit mit Gewaltmonopol. Die Demokratie wird oben aufgesetzt, sie gibt dem abstrakten Staat, der "Staatlichkeit", nur einen besonderen Inhalt; sie wird nicht zur Selbstorganisation der Gesellschaft, sondern erhält nur die Funktion, staatliche Herrschaft zu legitimieren. Damit ist der Übergang zu den Begründungen der Legitimation auch des Gewaltmonopols jenseits der abstrakten Staatlichkeit gefunden.

Weniger eloquent findet sich dieser Begründungszusammenhang in vielen juridischen Räsonnements über das Gewaltmonopol. So kann man etwa lesen: „Dem staatlichen Gewaltmonopol entspricht die Friedenspflicht des Bürgers. Diese bindet ihn jedoch nur, wenn der Staat seinerseits für Frieden und Ordnung im Gemeinwesen sorgt. "20 Die Bindung der Bürger an die Friedenspflicht ergibt sich auch hier nicht aus einer wie immer gearteten demokratischen Legitimation der Gesetze, sondern aus der Friedensfunktion des Staates an sich. Das heißt aber nichts anderes, als dass eine Basislegitimation schon in der effektiven Ausübung von Herrschaft, die notwendig eine Ordnung herstellt, liegt. Diese aus dem Wesen des Staates begründete Friedenspflicht der Bürger und Ordnungsfunktion des Staates ist eine vordemokratische Konstruktion, die die unterschiedlichsten Staatsformen - also ebenso Diktaturen, Monarchie oder Oligarchie rechtfertigen, solange sie eine (rechtliche) Ordnung herstellen. An dieser Stelle will ich

16 Forsthoff, E.: Verfassungsprobleme des Sozialstaats, S. 50.

17 Schmitt, C.: Der Begriff des Politischen, S. 39 f.

18 Böckenförde, E-W.: Der Staat als sittlicher Staat (Berlin 1978), S. 13. Böckenförde wörtlich: "Alle Streitigkeiten und Konflikte zwischen den Einzelnen oder zwischen Gruppen innerhalb des Staates werden friedlich, d.h. ohne Anwendung physischer Gewalt und in rechtlich geordneten Verfahren ausgetragen. Das ist ein Grundelement staatlicher Ordnung. Auch in der politischen Auseinandersetzung und im politischen Machtkampf innerhalb des Staates findet keine Freund-Feind-Gruppierung statt, welche die Bereitschaft zur physischen Gewaltanwendung einschließt, sondern alle Gegensätze verbleiben auf dem Intensitätsgrad einer Gesellschaft, welche die Einbindung in die gemeinsame Friedensordnung nicht sprengt."

19 Böckenförde, E-W.: Die Zukunft politischer Autonomie. Demokratie und Staatlichkeit im Zeichen von Globalisierung, Europäisierung und Individualisierung, in: ders., Staat, Nation, Europa, S. 108.

20 Schulte, M.: Gefahrenabwehr durch private Sicherheitskräfte im Lichte des staatlichen Gewaltmonopols, in: DVBl. 1995, S. 130. 
zunächst nur mit Verweis auf die Ausführungen zu Hobbes feststellen, dass eine vordemokratische Legitimation des Gewaltmonopols auf diesem Wege nicht ausreicht.

\section{Herrschaft und Legitimation von Gewalt}

Folgen wir Webers Definition von Herrschaft als „Chance, für einen Befehl bestimmten Inhalts bei angebbaren Personen Gehorsam zu finden", dann wird durch die Ordnungsfunktion Herrschaft legitimiert, denn mittels des Gewaltmonopols besteht die zitierte Chance offenkundig. Anders formuliert: Über die Ordnungsfunktion ist nach dieser Konzeption eine Basislegitimation von Herrschaft erfolgt. Die zusätzliche, demokratische Legitimation hat dann keine andere Funktion, als das Legitimationsniveau zu erhöhen. An eine vordemokratische Bestimmung des Staates wird die demokratische Form als etwas Zusätzliches herangetragen. Demokratie erscheint als eine Organisationsform und eine Legitimationsform der Herrschaft, nicht als Versuch diese aufzuheben. Böckenförde formuliert dies ausdrücklich: "Die Errichtung der Demokratie... bedeutet nicht die Aufhebung politischer Herrschaft, sondern eine bestimmte Organisation dieser Herrschaft. Staatsgewalt und die mit ihr gegebene Herrschaft von Menschen über Menschen bleibt auch in der Demokratie bestehen und wirksam, löst sich... nicht im herrschaftsfreien Diskurs auf."21 Die Staatsgewalt und mit ihr das Gewaltmonopol wird in dieser Konzeption einer hierarchischen Demokratie gleichsam zweistufig legitimiert, erstens durch die Ordnungsfunktion und zweitens durch das demokratische Verfahren, das als Legitimationsverfahren im Prinzip auf den Wahlakt beschränkt wird. Um es zugespitzt zu formulieren: Das Legitimationssubjekt wird gewechselt, an die Stelle Gottes in der alten Formel ,von Gottes Gnaden“ tritt das Volk, dem aber jeder weitere Zugriff auf staatliche Entscheidungsprozesse und die Ausübung der Staatsgewalt versagt bleibt.

Mit der Legitimation der Herrschaft wechselt das Subjekt. Die Staatsgewalt wird zum Subjekt, während das Volk in die Rolle eines Objekts zurückfällt, das die nun ja legitimierte Staatsgewalt erleiden muss. Es bleibt nichts vom individuellen, kontraktuellen Standpunkt in der Rechtfertigung staatlicher Gewalt, der bei Hobbes deutlicher herausgearbeitet ist als in der hierarchischen Theorie der Demokratie. Der Vertragsschluss ist bei Hobbes Unterwerfungsvertrag, aber verlangt vom Subjekt, das sich damit gleichzeitig zum Objekt macht, die Zustimmung zur Unterwerfung unter die öffentliche Gewalt. Die individuelle Zustimmung der Herrschaftsunterworfenen verflüchtigt sich in der hierarchischen Demokratie im legitimen Wesen des Staates als Ordnungsmacht und dem Legitimationsakt, der in der Theorie keineswegs als Zustimmung der Adressaten zu der mit dem Recht verbundenen Befugnis zu zwingen konstruiert ist. Die Entfremdung der politischen Eliten von den Objekten der Herrschaft, wie sie zunehmend deutlich wird, ist aus der Perspektive der hierarchischen Demokratie, der Legitimationsketten möglicherweise ein empirisches, aber kein theoretisches, normatives Problem.

21 Böckenförde, E-W.: Demokratie als Verfassungsprinzip, in: ders., Staat, Verfassung, Demokratie, S. 297; ähnlich: Der Staat als sittlicher Staat, S. 16; des., Die Zukunft politischer Autonomie, a.a.O., S. 107. 


\section{Gewalt-Monopol und Marktordnung}

Aber der juridische Diskurs ist manchmal durchaus historisch informiert. „Die Herstellung von Sicherheit, die Erzwingung des Friedens," schreibt Christian Calliess unter Berufung auf Norbert Elias, ,,hatte aber auch einen ökonomischen Hintergrund.“ Der sich entwickelnde Handel, die Entstehung von Märkten und Ansätze einer Marktgesellschaft hatte zur Folge, dass die Sicherheit der Handelsrouten vor „Raubrittern und marodierenden Banden“ 22 gewährleistet werden musste, wozu die dezentralisierten feudalen Gewalten nicht in der Lage waren. Ein Flickenteppich von lokalen und regionalen Machthabern, die jeweils eigene Zollsysteme hatten, stand der Ausweitung des Handelsverkehrs ebenso im Weg. Weiter mussten Instanzen geschaffen werden, welche die Einhaltung der Handelsverträge garantieren. Handelspartner treten sich grundsätzlich als Gleiche gegenüber, die Vertragsbrüche nicht ahnden können. Kurz: Die Entwicklung einer kapitalistischen Marktgesellschaft drängt zu einer von den ökonomischen Akteuren und letztlich von der Gesellschaft getrennten, verselbstständigten und zentralisierten Staatsgewalt.

Hegel erfasst dies bei aller Geisterbeschwörung, bei der der Staat als ,der Gang Gottes in die Welt" “23 firmiert, vergleichsweise klar. Die bürgerliche Gesellschaft ist für ihn schon Marktgesellschaft, in der konkurrierende Subjekte auf dem Markt auftreten, eine Gesellschaft, die in unterschiedliche Interessen zerfällt. Um ein reibungsloses Miteinander der Subjekte der bürgerlichen Gesellschaft zu sichern, bedarf es der „Aufsicht und Vorsorge der öffentlichen Macht. ... Die verschiedenen Interessen der Produzenten und Konsumenten können in Kollision miteinander kommen ... so bedarf die Ausgleichung auch einer über beiden stehenden mit Bewusstsein vorgenommenen Regulierung. " Und weiter: Das „Recht, nicht betrogen zu werden, und die Untersuchung der Waren (muss) als ein gemeinsames Geschäft von einer öffentlichen Macht vertreten und besorgt werden.“24 Das überhöht Hegel dann zum „Prinzip der Subjektivität sich zum selbstständigen Extreme der persönlichen Besonderheit vollenden zu lassen.“ Diese Individualisierung wird aber erkauft mit der Vereinheitlichung durch den Staat. Auch Hegel kennt das Wesen, d.h. die staatliche Substanz, die den individuellen und besonderen Staaten vorausgeht. ${ }^{25}$ Die Substanz besteht in der Bildung einer Einheit, die das Allgemeine gegenüber der zersplitterten bürgerlichen Gesellschaft repräsentiert, gleichzeitig aber deren Zersplitterung, d. h. die Subjektivität der Marktteilnehmer respektiert und bestehen lässt. Hegel weigert sich, die Legitimation der Staatsgewalt vom Individuum zu denken, ${ }^{26}$ denn dies müsste ihn im Jahre 1820 notwendig zu einer demokratischen Rechtfertigung der Staatsgewalt führen. Gerade das will er nicht, weil er sich dann mit der preußischen Monarchie anlegen müsste. Also denkt er die Staatsgewalt gleichsam vom Markt her, der den Zusammenhang der bürgerlichen Gesellschaft herstellt, das Allgemeine der Besonderen bildet, über das der Staat wachen muss, das im Staat synthetisiert wird. So wird die sittliche Idee in der Wirklichkeit Staatsgewalt. Das Subjekt bleibt Marktsubjekt, aber wird nicht Staatsbürger oder wie Marx es interpretiert hat: es

22 Calliess, Ch.: Sicherheit im freiheitlichen Rechtsstaat, in: ZRP 2002, S. 1/2.

23 Hegel, G.W.F.: Grundlinien der Philosophie des Rechts, § 258, Zusatz.

24 Hegel, G.W.F.: Grundlinien der Philosophie des Rechts, § $235 \mathrm{f}$.

25 Hegel, G.W.F.: Grundlinien der Philosophie des Rechts, § 259 Zusatz

26 Hegel, G.W.F.: Grundlinien der Philosophie des Rechts, § 258. 
ist Bourgeois, aber nicht Citoyen. Dass diese Idee noch nicht für die Europäische Union als Marktgesellschaft aufpoliert wurde, scheint eher auf einen Verfall intellektueller Kapazität als auf einen Fortschritt des demokratischen Bewusstseins hinzudeuten.

\section{Formkritik}

Ebenso wie Hobbes weist die Hegelsche Staatstheorie in die Zukunft, wenn sie auch die Reaktion in Preußen nach den Karlsbader Beschlüssen rechtfertig. Es liegt auf der Hand, - mit Marx - den Fortschritt von den droit de bourgeois zu den droit de citoyen zu fordern. Die Verwirklichung der Subjektivität in der Zirkulationssphäre und ihre Reduzierung auf das nur besondere Objekt in der Sphäre des Staates weist den Weg zur dialektischen Aufhebung, zu gesellschaftlichen Organisationsformen, in der die Trennung überwunden ist, das Gewaltmonopol den Subjekten nicht als fremde Macht gegenüber tritt, sondern als Verwirklichung ihres Rechts oder als Selbstverwirklichung des selbst gesetzten Rechts.

Hier ist die Gleichsetzung von Recht und Gewalt als Rechtskritik wieder aufzugreifen. Die Absonderung und Verselbstständigung der öffentlichen Gewalt gegenüber der Gesellschaft, die Herausbildung des Gewaltmonopols tritt - wie gesehen - historisch auf mit der Etablierung einer (früh)kapitalistischen Marktwirtschaft und ist gleichsam funktionale Bedingung für eine Marktgesellschaft. Marx dreht Hegel um - von mir aus auf die Füße: Der Staat, die Staatsgewalt ist nicht das Allgemeine, die Verallgemeinerung der Subjektivität, sondern das von der Gesellschaft besonderte. Der Staat ist ein besonderer Apparat, welcher der Gesellschaft als Gewaltapparat gegenüber tritt und der nicht etwa nur als neutrale Instanz die Marktordnung absichert, sondern neben den eigenen auch die mit der kapitalistischen Ordnung verbundenen Herrschaftsverhältnisse, insbesondere die Befugnis zur Aneignung der Produkte fremder Arbeit, garantiert. Der Staat mitsamt seinem Gewaltmonopol unterliegt ebenso wie das Recht der Formbestimmung der kapitalistischen Gesellschaft, es ist die Organisationsform des Kapitalismus. Deshalb geht es nicht darum sie zu legitimieren, sondern sie aufzuheben oder wie Engels es formuliert, den Staat absterben zu lassen. ${ }^{27}$ Eugen Paschukanis folgert weiter: "Den Übergang zum entwickelten Kommunismus stellt sich Marx folglich nicht als einen Übergang zu neuen Rechtsformen vor, sondern als ein Absterben der juristischen Form als solcher, als eine Befreiung von diesem Erbe der bürgerlichen Epoche." ${ }^{28}$ Kurz: Das Gewaltmonopol kann nicht legitimiert werden, auch nicht durch Recht.

\section{Droit des Citoyen: Aufhebung der Besonderung}

Nun mag es an meinem beschränkten, juristisch-bürgerlichen Horizont liegen, dass hier meine Vorstellungskraft an ein ende kommt. Auf Recht und die Befugnis zu zwingen, d.h. auch auf das Gewaltmonopol lässt sich nur verzichten, wenn man eine harmonische, konfliktfreie Gesellschaft unterstellt, in der keine konkurrierenden Interessen bestehen.

27 Engels, F.: Anti-Dühring, MEaW V, S. $307 \mathrm{f}$.

28 Paschukanis, E.: Allgemeine Rechtslehre und Marxismus (hrsg. von Klenner und Mamut) (Freiburg, Berlin 1991), S. 47, 61. 
Dafür reicht meine Fantasie nicht, auch nicht, wenn ich eine Aufhebung des kapitalistischen Klassenantagonismus antizipiere. Eine komplexe Gesellschaft ohne Konflikte ist wahrscheinlich auch kein Traum, sondern ein Albtraum. Die umfassende Versöhnung lässt sich metaphysisch als Nirwana denken, schwerlich aber als gesellschaftliche Wirklichkeit - auch nicht in der konkreten Utopie einer Gesellschaft, welche „die Produktion auf Grundlage freier und gleicher Assoziation der Produzenten neu organisiert", 29 so dass ,der enge bürgerliche Rechtshorizont ganz überschritten werden und die Gesellschaft auf ihre Fahne schreiben (kann): jeder nach seinen Fähigkeiten, jedem nach seinen Bedürfnissen!“ 30

Die Lösung auftretender Konflikte dem Recht des Stärkeren zu überlassen, muss wiederum als zivilisatorischer Rückschritt gewertet werden. Das kann nicht die Perspektive der Kritik an der Besonderung des Staates, d.h. am Herrschaftsanspruch und illegitimer Gewalt sein. Wenn das Gewaltmonopol die Negation gesellschaftlicher Gewalt ist, kann die Negation der Negation nur in der Rückverlagerung in die Gesellschaft, d.h. in der Aufhebung der Besonderung durch demokratische Teilhabe liegen. Demokratische Legitimation meint also nicht Legitimation von Herrschaft, sondern Aufhebung der Besonderung des Staates durch gleiche Teilhabe. Diese erfolgt mit Blick auf das Gewaltmonopol zunächst durch die gesetzliche Programmierung sowie richterliche und öffentliche Kontrolle der gesetzlich mit der Befugnis zur Gewaltanwendung ausgestatteten Institutionen.

Aber: Die Freiheit der Warenbesitzer setzt nicht nur die Absicherung des Marktgeschehens durch einen spezialisierten Gewaltapparat voraus, sondern produziert gleichsam notwendig auch einen spezialisierten Rechtsapparat. Das Marktgeschehen und letztlich die Marktgesellschaft funktioniert nur, wenn die sich herausbildende Staatsgewalt Bindungen unterworfen ist, nach berechenbaren Regeln handelt, die für den Marktteilnehmer oder genauer: für den Kapitalisten berechenbar sind. Die Rechenhaftigkeit der kapitalistischen Geschäfte verlangt ein berechenbares Recht. Das ist nicht Marx, sondern Weber. Die formale Rationalität des kapitalistischen Geistes und Marktes führt zu homologen Phänomenen auch im Bereich des Rechts und des Staates. Das ist noch nicht mehr als der formale Rechtsstaat, der oben diskutiert wurde. Trotz der Berechenbarkeit, also trotz des Rechtscharakters kann das Recht grob ungerecht und mit brutaler Unterdrückung verbunden sein.

Marx ist wieder weiter als Weber, wenn er spottet: „Die Sphäre der Zirkulation oder des Warenaustausches, innerhalb deren Schranken Kauf und Verkauf der Arbeitskraft sich bewegt, war in der Tat ein wahres Eden der angebornen Menschenrechte. Was allein hier herrscht, ist Freiheit, Gleichheit, Eigentum und Bentham. Freiheit! Denn Käufer und Verkäufer einer Ware, z.B. der Arbeitskraft, sind nur durch ihren freien Willen bestimmt. Sie kontrahieren als freie, rechtlich ebenbürtige Personen. Der Kontrakt ist das Endresultat, worin sich ihre Willen einen gemeinsamen Rechtsausdruck geben. Gleichheit! Denn sie beziehen sich nur als Warenbesitzer aufeinander und tauschen Äquivalent für Äquivalent. Eigentum! Denn jeder verfügt nur über das Seine. Bentham! Denn jedem von den beiden ist es nur um sich zu tun. Die einzige Macht, die sie zusammen und in ein Verhältnis bringt, ist die ihres Eigennutzes, ihres Sondervorteils,

29 Engels, F.: Der Ursprung der Familie, MEW Bd. 21, S. 168.

30 Marx, K.: Kritik des Gothaer Programms, MEW Bd. 19, S. 21. 
ihrer Privatinteressen. Und eben weil so jeder nur für sich und keiner für den andren kehrt, vollbringen alle, infolge einer prästabilierten Harmonie der Dinge oder unter den Auspizien einer allpfiffigen Vorsehung, nur das Werk ihres wechselseitigen Vorteils, des Gemeinnutzens, des Gesamtinteresses." 31

Der formale Rechtsstaat des berechenbaren Warentausches wird hier inhaltlich aufgefüllt durch die zentralen Menschenrechte der liberalen Epoche: Freiheit, Gleichheit und Eigentum. Anders formuliert: Die Marktordnung ist nicht nur mit der Rechtsform verbunden, sondern auch mit spezifischen Inhalten, mit Menschenrechten, die sich in der liberalen Epoche zumindest für das Marktgeschehen durchsetzen. Kann nun nicht dieser material verstandene Rechtsstaat, der Freiheit, Gleichheit und Eigentum der Warenbesitzer garantiert, zusammen mit der Ordnungsfunktion das Gewaltmonopol rechtfertigen oder anders: Kann man nicht auf Demokratie zur Rechtfertigung des Gewaltmonopols verzichten: reicht für die Basisrechtfertigung nicht der materiale Rechtsstaat? Reichen die Droit de Bourgeois, d.h. die Menschenrechte ohne Meinungs- und Koalitionsfreiheit i.w.S. und ohne soziale Rechte, nicht zur Rechtfertigung des Gewaltmonopols?

Die Rechtsform ist in dieser Deutung und - wie gesehen mit Hegel - mit der Konstituierung nicht nur des Rechtssubjekts, sondern des Subjekts überhaupt verbunden anders gesagt, die Zuweisung von Rechten an die Marktteilnehmer gilt als zumindest erster Schub der Individualisierung, der mit der Herausbildung der Moderne oder der Entstehung kapitalistischer Marktgesellschaften verbunden ist. Mit der Freisetzung der Märkte, die ja keineswegs die natürliche oder ursprüngliche Form der Vergesellschaftung darstellen, ${ }^{32}$ ist die Freisetzung der Subjektivität verbunden, die aber nicht teilbar ist. Besser: Die Teilung, die Aufspaltung in Bourgeois und Citoyen ist normativ nicht zu rechtfertigen. Es gilt das gleiche wie für Hobbes Vertragstheorie: Die Anerkennung des Zustimmungserfordernisses zum Gesellschaftsvertrag führt normativ zur Forderung nach einem Zustimmungserfordernis zu allen Akten der Herrschaft. Das lässt sich analytisch als Dilemma der bürgerlichen Konstitution charakterisieren: Die mit der Anerkennung der Marktrechte verbundene Herrschaftsform verweist auf die Ausweitung dieser Rechte auf die politische Ebene und unterminiert eben diese Herrschaftsform. Wenn die oberen die Melodie von Freiheit und Gleichheit pfeifen, können sie sich nicht wundern, wenn die unteren anfangen, die Verhältnisse danach zum Tanzen zu bringen.

Der liberale Unternehmer will in Ruhe seinen Geschäften nachgehen und möchte diese und sein Eigentum vor Übergriffen durch den Adel und korrupte Beamte ebenso geschützt sehen wie vor dem „Pöbel“. Religionsfreiheit, Vertragsfreiheit, Gleichheit vor dem Gesetz, Eigentum und Sicherheit ${ }^{33}$ charakterisieren die Menschenrechte der liberalen Epoche, die mit spezifischen Klasseninteressen korrelieren, während die Grundrechte, welche demokratische Teilhabe absichern, notwenigerweise gleichursprünglich mit dieser sind. Die Legitimation des Gewaltmonopols über den liberalen Rechtsstaat ist so historisch als wirksamer, aber ideologischer Versuch zu werten, demokratische Forderungen einzuhegen, d.h. mit der Begrenzung auf die droit de Bourgeois die mit

31 Marx, K.: Das Kapital I, MEW Bd. 23, S. 189 f.

32 Polanyi, K.: The Great Transformation. Politische und ökonomische Ursprünge von Gesellschaften und Wirtschaftssystemen (1944), Frankfurt 1978, passim.

33 In der französischen Bedeutung dieses Menschenrechts, die Sicherheit vor Verhaftungen meint. 
diesen verbundene soziale Formation oder die Produktions- und Herrschaftsverhältnisse zu sichern.

Ich versuch es noch einmal etwas klassischer - mit Kant: Der Grund dafür, dass die Befugnis zu zwingen und Freiheit vereinbar sind, ist für Kant die Lauterkeit des Ursprungs des Gesetzes. Besser gesagt: Nur der lautere Ursprung des Gesetzes rechtfertigt die Möglichkeit des Zwangs. „Nun hat aber die republikanische Verfassung außer der Lauterkeit ihres Ursprungs, aus dem reinen Quell des Rechtsbegriffs entsprungen zu sein, noch die Aussicht in die gewünschte Folge, nämlich den ewigen Frieden; wovon der Grund dieser ist. - Wenn (wie es in dieser Verfassung nicht anders sein kann) die Beistimmung der Staatsbürger dazu erfordert wird, um zu beschließen, ob Krieg sein solle, oder nicht', so ist nichts natürlicher, als dass, da sie alle Drangsale des Krieges über sich selbst beschließen müssten (...), sie sich sehr bedenken werden, ein so schlimmes Spiel anzufangen." ${ }^{34}$ Die Friedensordnung ist der demokratischen Gesetzgebung nicht vorgelagert, sondern - das sieht Kant völlig richtig - folgt aus ihr, nach außen, weil der Krieg für die Bürger in jeder Beziehung Unannehmlichkeiten verursacht. Die Bevölkerung ist gegen den Afghanistan Einsatz, was die politische Elite aber nicht sonderlich schert.

Kommen wir zurück zu Kant: die Beistimmung, „ob Krieg sein solle oder nicht““, ist gleichsam ein Unterfall der Beistimmung zu allen gesetzgeberischen Akten: „Vielmehr ist meine äußere (rechtliche) Freiheit so zu erklären: sie ist die Befugnis, keinen äußeren Gesetzen zu gehorchen, als zu denen ich meine Beistimmung habe geben können. " 35 Die Befugnis zu Zwingen ist nur deshalb mit der Freiheit vereinbar, weil sie auf einem allgemeinen Gesetz basiert, dem diejenigen ihre Beistimmung geben konnten, die im Zweifel gezwungen werden können. Die Begründung, warum die Zustimmung nicht zu Unrecht führen kann, lautet: „Die gesetzgebende Gewalt kann nur dem vereinigten Willen des Volkes zukommen. Denn da von ihr alles Recht ausgehen soll, so muss sie durch ihr Gesetz schlechterdings niemand unrecht tun können. Nun ist es, wenn jemand etwas gegen einen anderen verfügt, immer möglich, dass er ihm dadurch unrecht tue, nie aber in dem, was er über sich selbst beschließt (denn volenti non fit iniuria). Also kann nur der übereinstimmende und vereinigte Wille aller, so fern ein jeder über alle und alle über einen jeden ebendasselbe beschließen, mithin nur der allgemein vereinigte Volkswille gesetzgebend sein. “" ${ }^{36}$ Rousseau formuliert das ganz ähnlich: „Da die Bürger durch den Gesellschaftsvertrag alle gleich sind, können auch alle vorschreiben, was alle tun müssen, während keiner das Recht hat, von einem anderen etwas zu fordern, was er nicht selber macht." 37

\section{Legitimation-Legitimität}

Sind diese Anforderungen nicht zu hoch? Können Regime durch die Herstellung von Frieden und sozialer Sicherheit nicht ebenfalls Legitimität erlangen und das Gewaltmonopol legitim beanspruchen? Notwendig ist eine Unterscheidung zwischen Legiti-

34 Kant, I.: Zum ewigen Frieden, Erster Definitivartikel, S. 127 f.

35 Kant, I.: Zum ewigen Frieden, Erster Definitivartikel, S. 126.

36 Kant, I.: Metaphysik der Sitten, § 46, S. 432.

37 Rousseau, J.J.: Vom Gesellschaftsvertrag, S 132. 
mität und Legitimation. Die Frage nach der Legitimation des Gewaltmonopols meint nicht die Frage nach der faktischen Akzeptanz. Die Legitimation ist ein Prozess, der normativ gedacht werden muss, als Prozess der Ermächtigung. So klingt die zweite Bedeutungsvariante von Legitimation an: Bevollmächtigung, Ausgewiesenheit. An den Vorgang der Legitimation, das Gewaltmonopol auszuüben, sind notwendig hohe normative Anforderungen zu formulieren. Weber hat mit den drei Typen legitimer Herrschaft dagegen auf die faktische Akzeptanz abgestellt, die der charismatischen Herrschaft möglicherweise Legitimität im Sinne faktischer Akzeptanz im Sinne von Anerkennung, Zustimmung oder nur Erduldung einer Herrschaft verleiht, während die Legitimation, der Prozess, der dem charismatischen Führer zur Legitimität verhilft, im Dunkeln bleibt. Weber will und kann ihn nicht normativ rechtfertigen.

In der Demokratietheorie wird der Unterschied zwischen demokratischem Output und Input gemacht - was allerdings zu erheblichen Verwirrungen führen muss. Ein politischer Output, der Interessen oder Werten der Herrschaftsunterworfenen entgegen kommt, kann zur Akzeptanz in obigen doppelten Sinne der nicht legitimierten Herrschaft führen. Sie erscheint dann im Weberschen Sinne als legitime Herrschaft, aber eben nicht als legitimierte Herrschaft - sie besitzt also keine Legitimation. Die Legitimation, der Vorgang des Legitimierens ist nur als demokratisches Input zu denken - nur dieser Aspekt bezieht sich auf die Demokratie. Bezieht man den Output in den Begriff der Demokratie ein, kommt man unweigerlich zu der Konsequenz, dass möglicherweise auch der gute Monarch oder Diktator demokratisch sind. Sie besitzen schließlich faktische Akzeptanz. Aber es widerspricht offensichtlich der traditionellen ${ }^{38}$ und der zeitgenössischen Begriffsbestimmung der Demokratie deutlich. Der Vorgang der Legitimation kann sich also nicht auf das Output beziehen: den guten Monarchen oder die gute Ordnung des Rechtsstaates, sondern nur auf den demokratischen Input. Ob schließlich das Fehlen eines demokratischen Inputs die faktische Akzeptanz oder die Legitimität eines Systems untergräbt, ist eine empirische und historische, aber keine normative Frage.

\section{Widerstand und Recht}

Die Forderung nach einer Zustimmung aller Gleichen zu allgemein verbindlichen Entscheidungen führt offenkundig zu Problemen mit der Mehrheitsregel, die hier aber ebenso wenig diskutiert werden sollen wie Kants enge Definition des Staatsbürgers als wirtschaftlich selbstständig, was Frauen seinerzeit logisch ausschloss. Interessant ist noch die Frage der Repräsentation. Die Zustimmung wird von allen gefordert. Rousseau lehnt Repräsentation rundheraus ab, Kant fordert sie ein: „Alle wahre Republik aber ist und kann nichts anders sein, als ein repräsentatives System des Volks, um im Namen desselben, durch alle Staatsbürger vereinigt, vermittelst ihrer Abgeordneten (Deputierten) ihre Rechte zu besorgen. “39 Wir wissen inzwischen, dass die Repräsentation die Zustimmung aller und selbst der Mehrheit nur imaginiert. Zwischen Repräsentanten und Repräsentierten können gewaltige Differenzen klaffen. Die Aufhebung der Besonde-

38 Gemeint ist die Tradition von Aristoteles bis Kant.

39 Kant, I.: Die Metaphysik der Sitten, Kant-Werke Bd. 8, S. 464. 
rung des Staates lässt sich offenbar nicht als abgeschlossene Installation gesellschaftlicher Institutionen denken, sondern bedarf beständig demokratischer Prozesse der Erneuerung der Rückbindung staatlicher Willensbildung an die Gesellschaft.

Aber es steht noch etwas anderes im Raum: Kant lehnt jedes Widerstandrecht ab, das einmal errichtete Gewaltmonopol kann nur evolutionär beseitigt oder legitimiert werden. „Der Grund der Pflicht des Volks, einen, selbst den für unerträglich ausgegebenen Missbrauch der obersten Gewalt dennoch zu ertragen, liegt darin: dass sein Widerstand wider die höchste Gesetzgebung selbst niemals anders, als gesetzwidrig, ja als die ganze

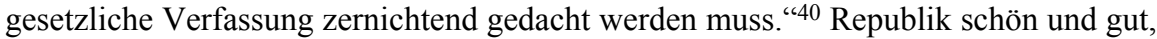
aber sie muss von oben kommen.

Das Grundgesetz geht weiter: Es kennt ein Widerstandsrecht gegen jeden, der es unternimmt, die grundgesetzliche Ordnung zu beseitigen. Zu dieser gehört das Demokratieprinzip, d.h. die Rückbindung der staatlichen Gewalt an die gesellschaftliche Willensbildung. Um keine Missverständnisse aufkommen zu lassen: Ein Widerstandsrecht ist extralegal und kann sicher nicht vom BVerfG verordnet werden. Das heißt aber erstens: Ein Widerstandsrecht gibt es - nach dem Willen des GG - nicht erst, wenn das Gewaltmonopol sich von rechtsstaatlichen Bindungen löst, sondern ,schon“ dann, wenn die demokratische Ordnung beseitigt werden soll. Eine „Gehorsamspflicht“" kennt das GG nur gegenüber demokratischer Gesetzgebung - unser Ergebnis lässt sich also positivistisch bestätigen.

Zweitens: Wenn die Staatsgewalt nun das Gewaltmonopol versteht als Befugnis, der Gewalt freien Lauf zu lassen. Wenn die Repräsentanten dies anschließend gegen die Repräsentierten rechtfertigen unter Berufung auf die Parlamentsentscheidung, die keineswegs repräsentierend ist, wird es schwierig mit der Friedenspflicht. Die Legitimität des Gewaltmonopols entspringt nicht dem ewigen Frieden des Friedhofs; der innere Frieden bedarf der gelungenen Repräsentation, eben der Aufhebung der Besonderung des Staates. Ein Schelm, wer an Stuttgart oder Gorleben denkt. Aktuell zeigen die Menschen in den arabischen Staaten, dass die Friedhofsruhe mit Gewaltmonopol eines Autokraten auf Dauer keineswegs dem Aufstand, der das Gewaltmonopol schleifen muss, vorgezogen wird und werden sollte - Ausgang offen.

40 Kant, I.: Die Metaphysik der Sitten, Kant-Werke Bd. 8, S. 440. 